\title{
Supporting Information: A review of imputation strategies for isobaric labeling-based shotgun proteomics
}

\author{
Lisa M. Bramer ${ }^{1}$, Jan Irvahn², Paul D. Piehowski, Karin D. Rodland ${ }^{3}$, Bobbie-Jo M. Webb- \\ Robertson ${ }^{3 *}$ \\ ${ }^{1}$ Computing Analytics Division, Pacific Northwest National Laboratory, Richland, WA, USA \\ 2 Boeing, Seattle, WA, USA \\ ${ }^{3}$ Biological Sciences Division, Pacific Northwest National Laboratory, Richland, WA, USA \\ *Corresponding Author: 902 Battelle Blvd, Richland, WA 99354, bj@pnnl.gov \\ Table of Contents
}

Figure S1. Correlation between mean intensity and percentage of missing values for simulated data Figure S2. Median proportion of peptides sampled from each intensity bin for simulated data Figure S3. Distribution of RMSE values for each imputation method Figure S4. Mean RMSE values for each imputation method Figure S5. Rank of imputation methods based on mean RMSE values Figure S6. Standard deviation of RMSE values, for each imputation method Figure S7. Distribution of false positive rates for each imputation method Figure S8. Cross-validation AUC distributions for two datasets. Imputation Code. $\mathrm{R}$ code for implementation of imputation methods. 


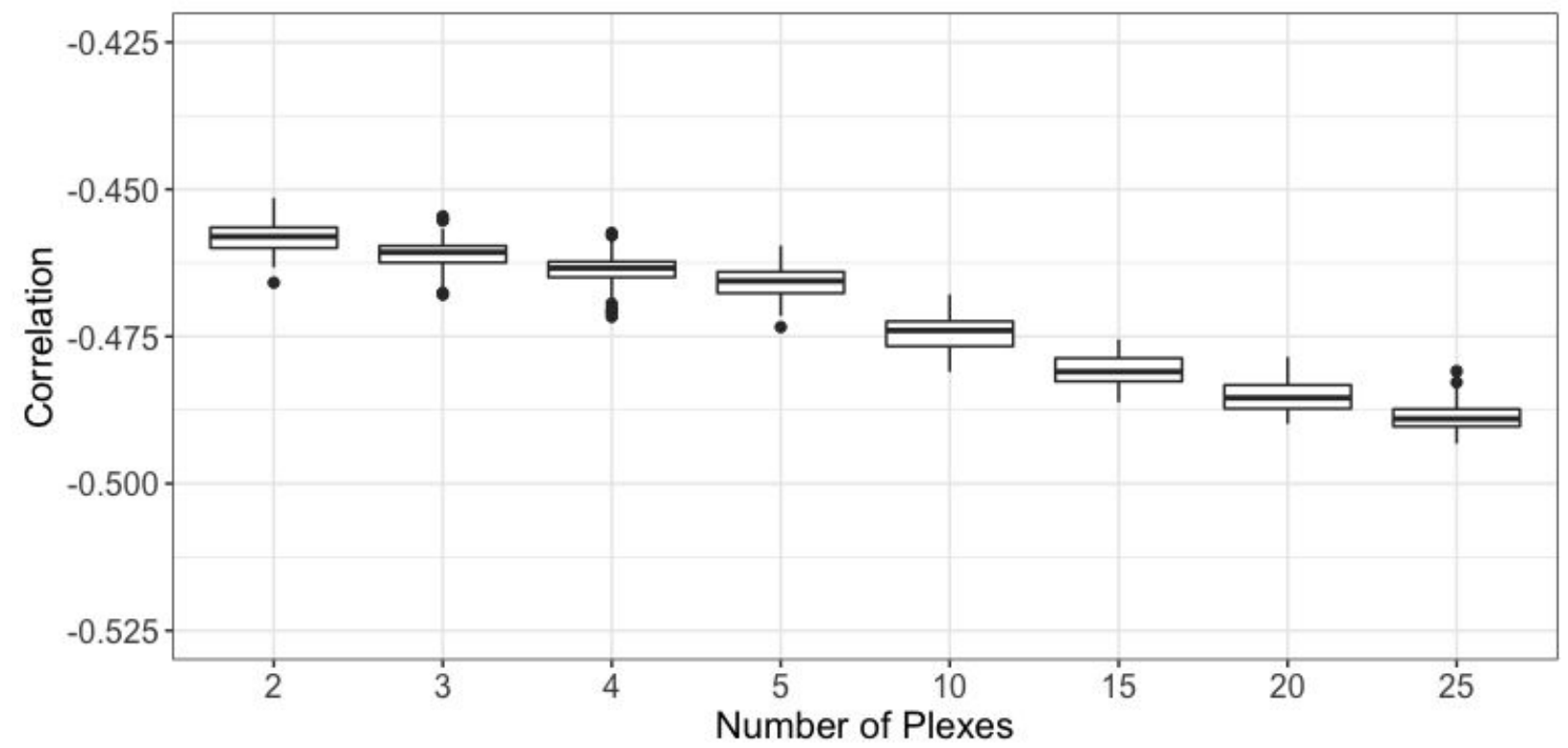

Figure S1: Observed correlation distributions between log2 mean peptide abundance (before normalization) with percentage of missing data, by the number of plexes, for the 3200 simulated datasets.

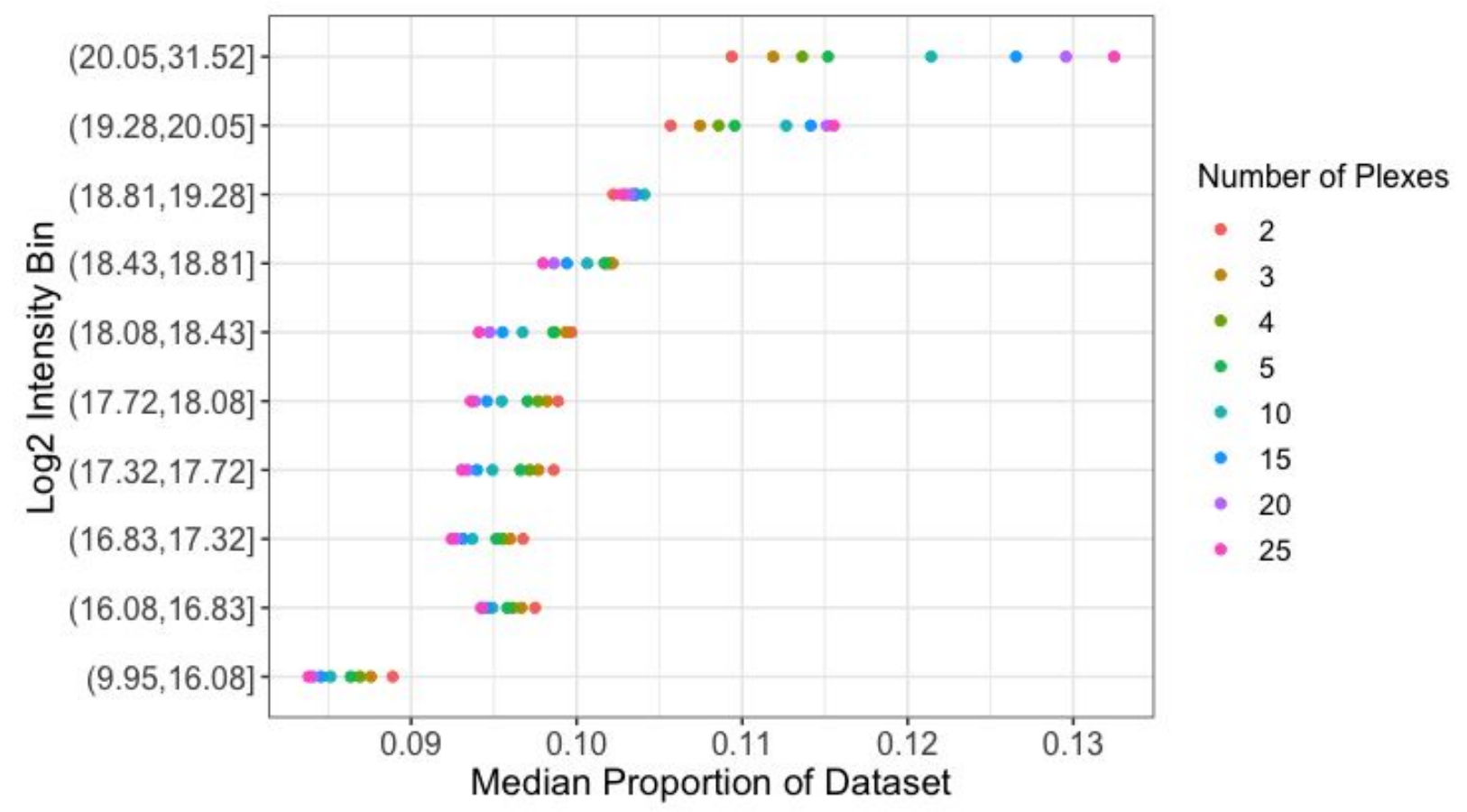

Figure S2: Median proportion of peptides sampled from each intensity bin, by number of plexes, across the 3200 simulated datasets. 


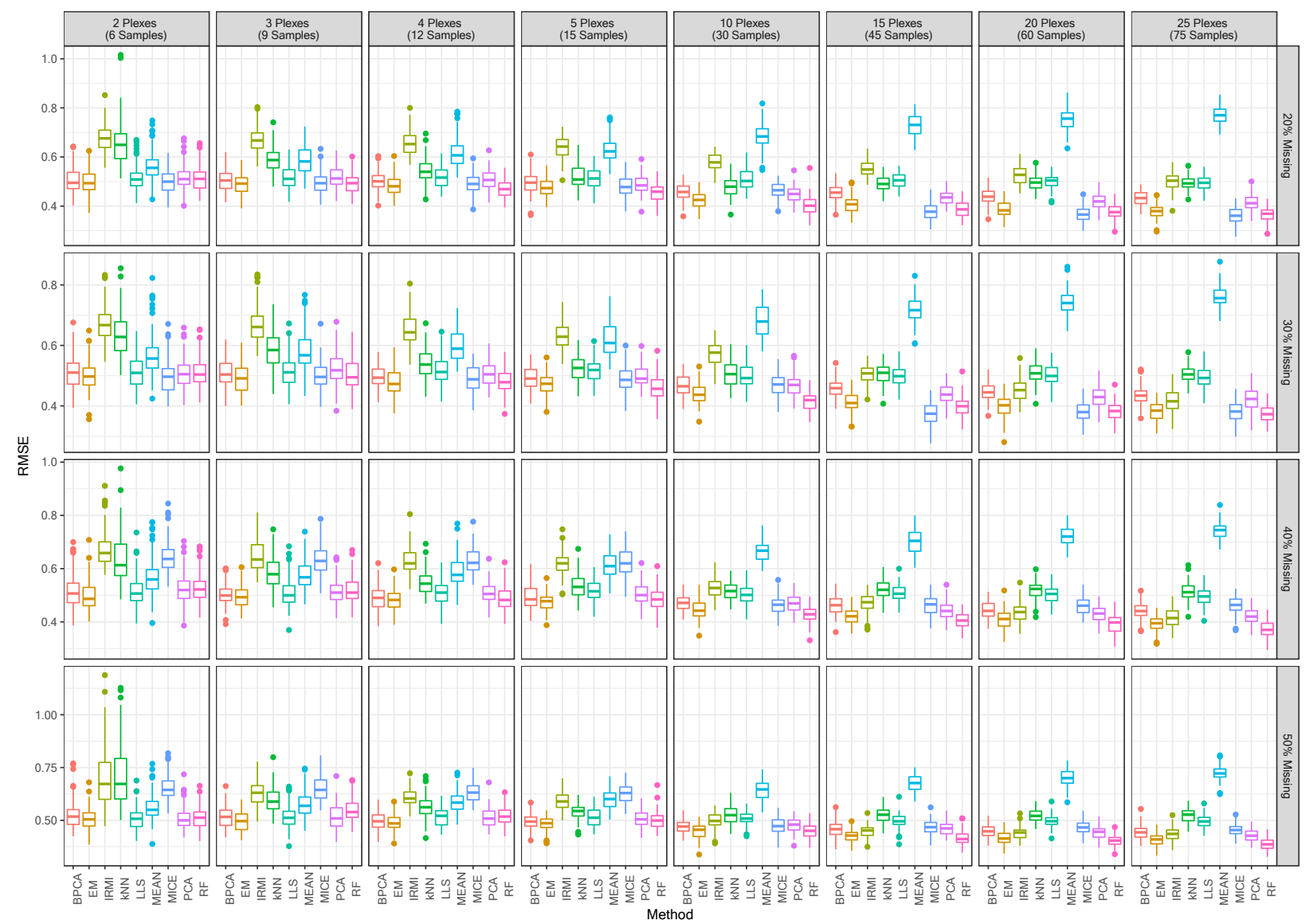

Figure S3: Distribution of RMSE values for each imputation method by percentage of missing data and number of plexes. 

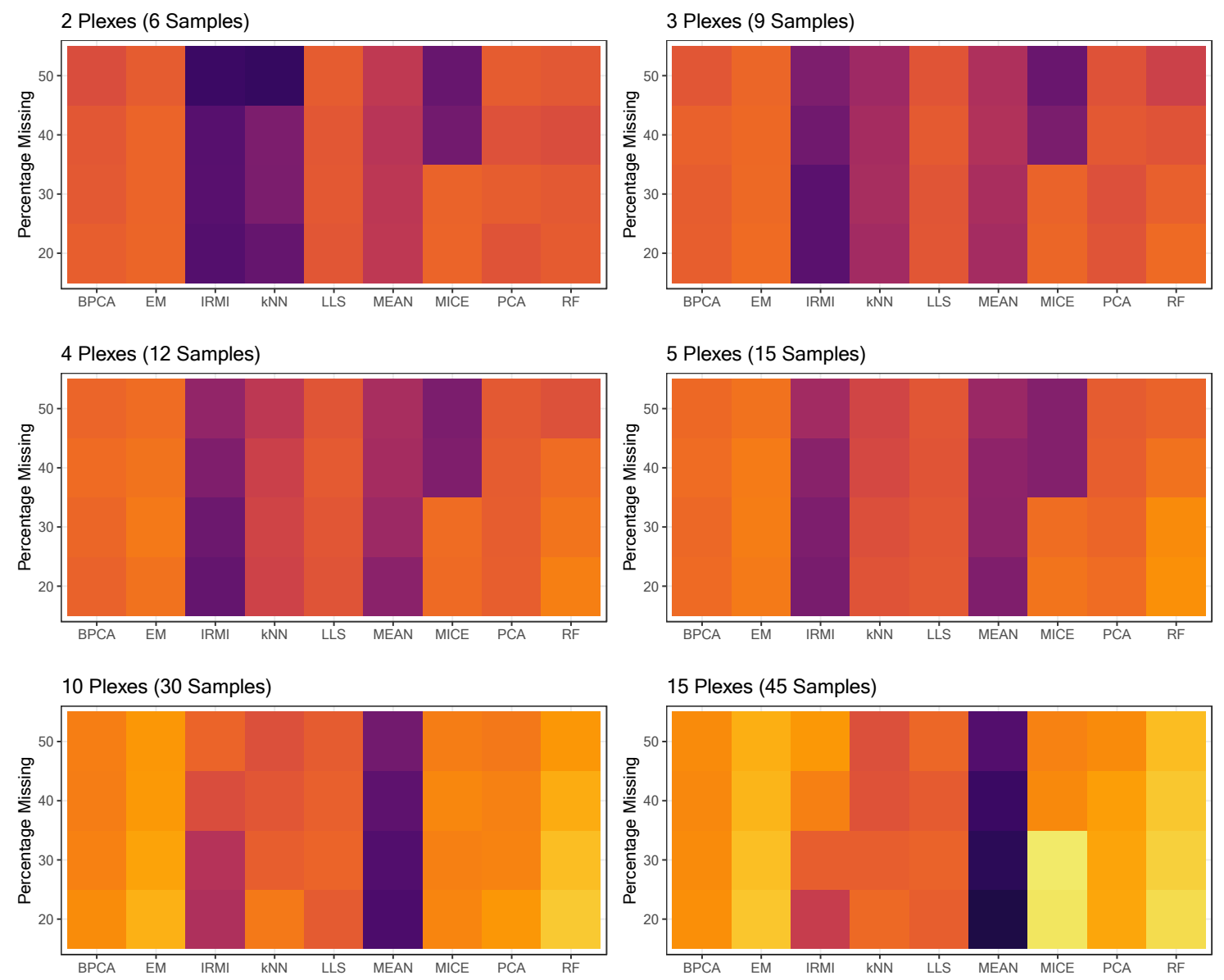

Mean RMSE

20 Plexes (60 Samples)
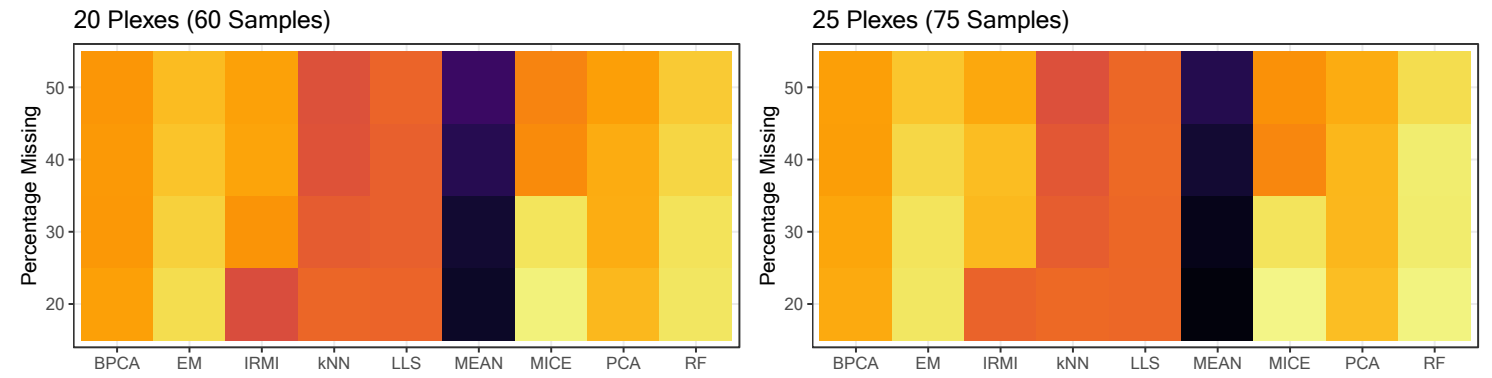

Figure S4: Mean RMSE values for each imputation method by percentage of missing data and number of plexes. 


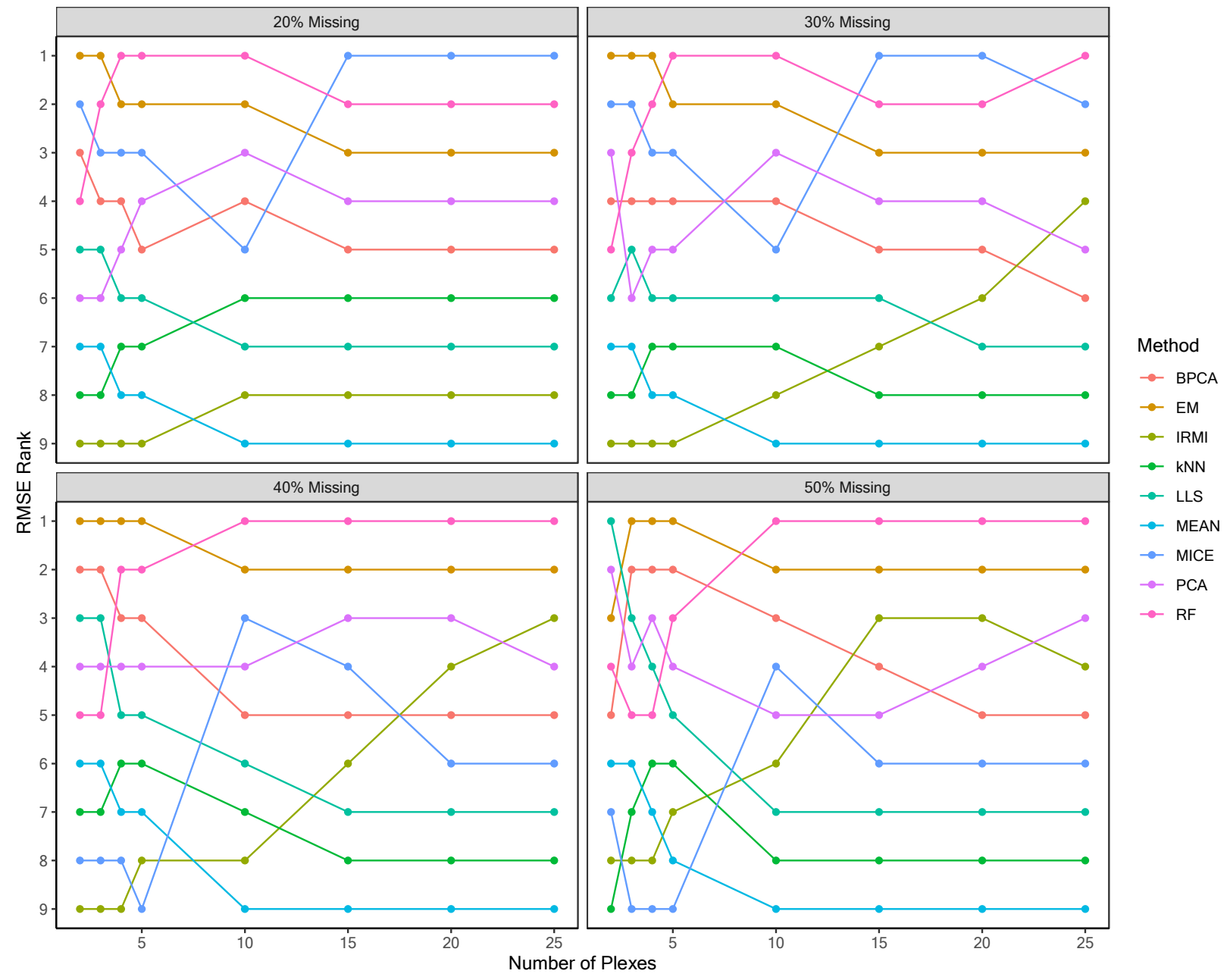

Figure S5: Rank of imputation methods based on mean RMSE values by the number of plexes for each percentage of missing values tested. Rank of 1 indicates imputation method with smallest mean RMSE. 


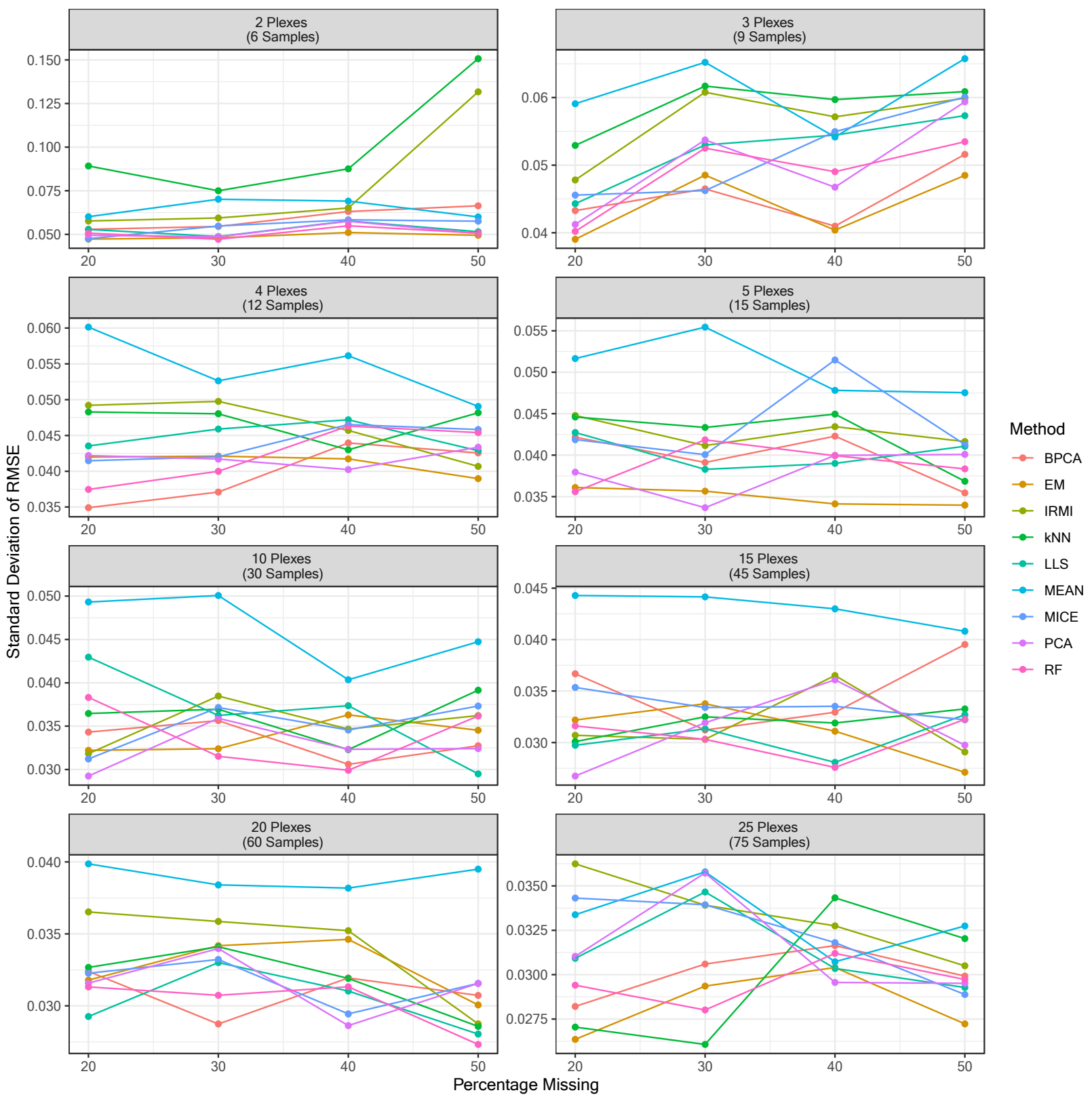

Figure S6: Standard deviation of RMSE values, for each imputation method, by percentage missing data and number of plexes. 


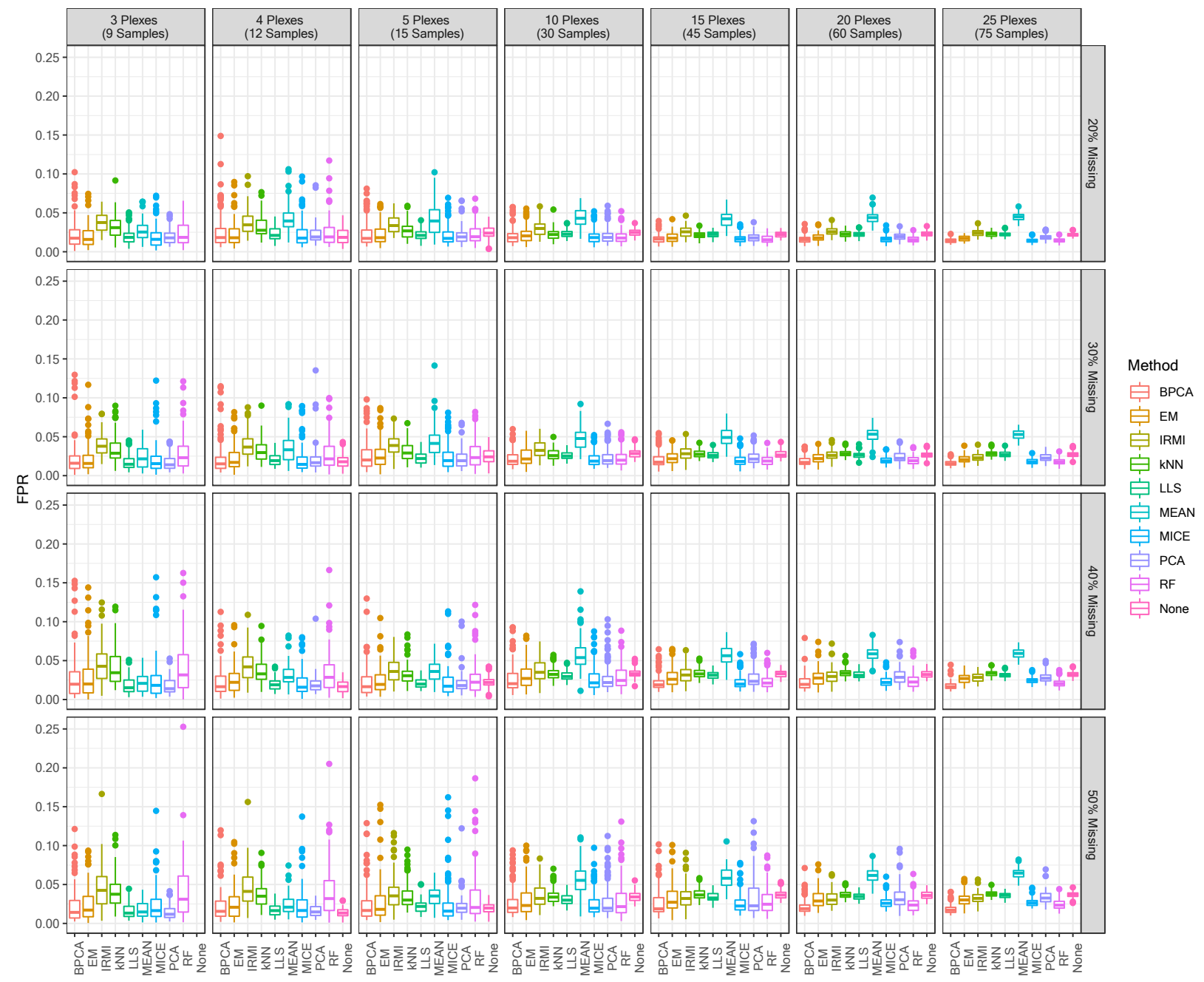

Figure S7: False positive rates for each imputation method by percentage of missing data and number of plexes. 


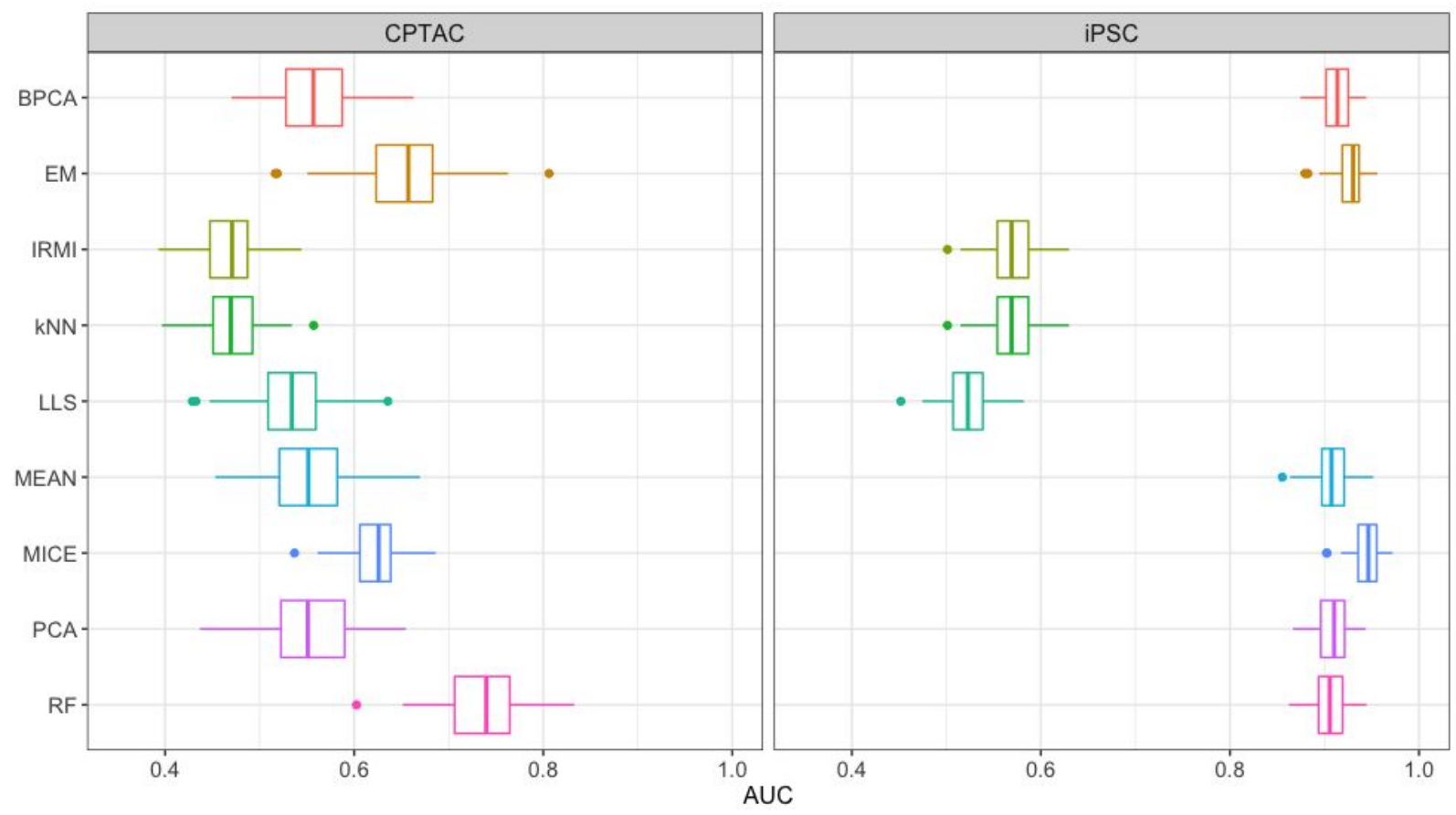

Figure S8: Cross-validated classification AUC under the ROC curve distributions, over 100 repetitions of 5-fold cross-validation, for each imputation method by dataset. 


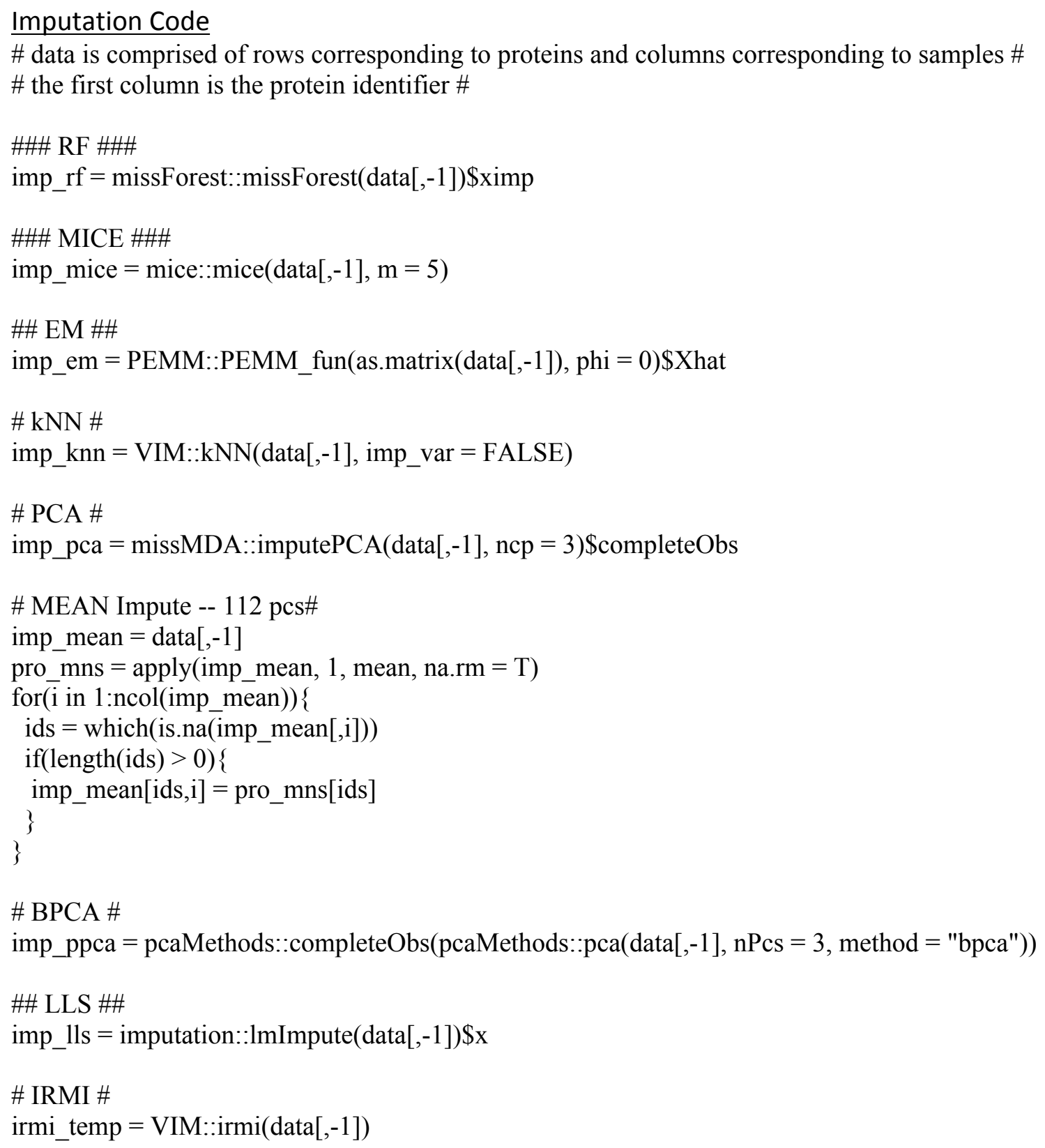

